$\lambda_{3} / 1 / 3\left(\lambda_{1},+\lambda_{2}+\lambda_{3}\right)\left(\lambda_{3}\right.$ is assumed to be the smallest eigenvalue). So we see that for jets we should have $S<<1$ (i.e. near zero) and $S \cong 1$ for events with large multiplicity and particles isotropically distributed in phase space.

\section{FREE QUARKS?}

Although from about 1965 physicists have been looking for fractionallycharged single quarks, there is not, as yet, any conclusive evidence as to their existence. At present the search goes on with determination, buoyed up by the fact that at least one of these quarks should be absolutely stable because of charge conservation. Methods mentioned here are not applicable if quarks are unstable and integrally charged (like the ones in the Pati-Salam model). The main searches are being done:- (i) with accelerators, (ii) in cosmic rays, and (iii) in stable matter. The method covered in the Conference was of the third kind and is a "descendent" of the Millikan's oil drop experiment. (see $E N, 1977,7 / 8$, p. 7).

The main improvements in the method since they first started ( 1965) are:-

(i) increasing the mass of the levitated object from

$\mathrm{m} \cong 2 \times 10^{-9} \mathrm{~g}$ to $\mathrm{m} \cong 10^{-4}-2 \times 10^{-4} \mathrm{~g}$ (Cf. Millikan's: $m \cong 10^{-11} \mathrm{~g}$ ) giving thus more chance of observing quarks, and (ii) using the feedback levitation method to keep the test sphere of iron in a fixed horizontal plane.

\section{Strong Interaction Dynamics}

The dynamics of strong interactions has puzzled physicists ever since it was postulated. Due to the very large coupling constant one cannot construct a perturbative theory similar to quantum electrodynamics which agrees so well with experiment, at least at presently available energies. Physicists have invented and used many different techniques in their attempt to understand strong interactions. Among these are dispersion theory, S-Matrix theory, "Regge theory" and Duality. None is adequate to explain all the data

Recently, however the foundations of a quantum field theory (Q.F.T.) of strong interactions have been set, namely "quantum chromodynamics" (Q.CD)

Q.CD is a non-Abelian Q.F.T. of quarks that is locally gauge invariant with respect to (exact) $\mathrm{SU}(3)$ symmetry. The coloured quarks interact via the exchange of coloured vector mesons (i.e. the 8 gluons) corresponding to the 8 generators of $\mathrm{SU}(3)$ colour. Note that in this theory the dynamics depends (or "resides") on colour alone and flavour merely serves as a quark label.

Non-Abelian gauge field theories like Q.CD have some unusual properties. For example:-

(i) the effective interaction between the quarks decreases as the energy and momentum transfer increase. As these variables in- crease asymptotically to infinity, the theory approaches a free field theory.

This property is the Asymptotic Freedom;

(ii) the theory has infrared divergencies. It has been speculated that this property of the theory leads to the confinement of all coloured states, including quarks and gluons. Such confinement is called "infrared slavery"! It is not yet known (conclusively) whether Q.CD does confine quarks and gluons. (A task for the future).

As quarks have not been observed so far, physicists have been trying hard to construct phenomenological models in which quarks are permanently confined. Examples are "the string model(s)" and the Bag models. Bag models have proved very successful, e.g., in explaining the hadronic mass spectrum and the Regge trajectories.

To conclude we compare and contrast Q.ED and Q.CD very briefly:-

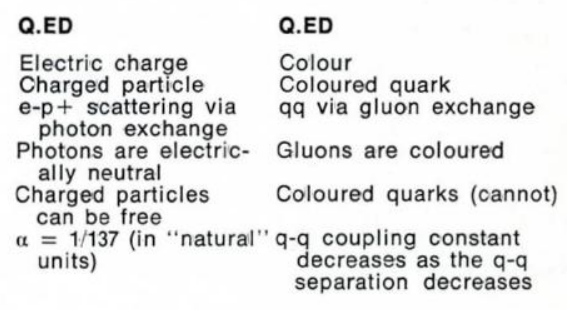

Majid Rahnama

\title{
Surfaces and Experimental Techniques
}

The extent of current interest in surface physics is indicated by the decision to hold a symposium of five halfdays on the subject, of which one was held in conjunction with the synchrotron radiation group in view of the impact that this new diagnostic source has had. Particular emphasis was placed on the study of semiconductors and surface magnetism.

In the field of semiconductors a great deal of work has been done on relating the electronic properties of the surface and interface to structural characteristics particularly in GaAs. The electronic properties of an ideal heterojunction have been analysed and models of particular systems developed. Imperfections are of great significance in real situations, arising both from intrinsic defects and from impurities present in minute concentrations. Defects have a dramatic ef- fect on the electronic properties of surfaces and on the first stages of oxidation. This is of great importance for example in the preparation of substrates for molecular beam epitaxy, but in recent times it has proved possible to produce monocystalline thin films with precise control of uniformity, thickness and doping profile.

Much has been accomplished in measuring and understanding surface magnetism effects, but in ferromagnetic materials the existence of reduced magnetization near the surface the "dead layer" still remains unexplained.

On the experimental side, the techniques now at the command of the surface physicist are remarkably varied, and provide such a detailed description of surface phenomena it becomes proper to ask how this information can be used. Classical methods include low energy electron diffraction and neutral thermal beam diffraction, the latter providing new information about the gas-surface interaction. With medium energy ion scattering, by using a $100 \mathrm{keV}$ proton beam and measuring the blocking pattern from Rutherford back scatter information is derived on the planar spacing of the first two layers and the location of absorbed atoms. Surface atoms are located with a precision of $0.02 \AA$. High resolution electron loss spectroscopy is particularly well suited to study vibrational spectra of atoms and molecules absorbed on the surfaces of single crystals, and offer a unique possibility of investigating ordered adsorbate structures.

Newly on the scene is infrared photoacoustic spectroscopy whereby an acoustic wave is set up by absorption of a modulated light beam. The me- 
thod seems to offer considerable promise for the study of optical and absorption spectra of, particularly, non-transparent solids and liquids. High energy electron diffraction theory has been refined to take care of inelastic effects so that this method is now a more valuable tool to the experimenter. Low energy, high resolution photoemission techniques have been used to demonstrate, for example, the change in the electronic structure caused by melting. Then we have polarized electron emission, Auger spectroscopy and the range of measu- rements that can be made with synchrotron radiation varying from the use of X-ray absorption techniques to high angular resolution of UV photoemission which permits the direct determination of the electronic band structure. In this general area of photoemission processes, important advances have also been made in the theory which allows a better understanding of the significance of experimental determinations.

\section{Françoise Houzay and Lubomir Skala}

\section{Exhibition}

Centrally placed in the exhibition mounted at York was a series of panels explaining the basic principles and the characteristics of synchrotron radiation, tracing its development as an experimental technique from the time the effect was first observed.

Because the radiation energy can be so precisely controlled and fo- cused, medical radiography and, in particular, tomography, are bound to benefit. Using small-angle scattered radiation at, say, $140 \AA$, provides a powerful means for the study of biological substances and has already furnished us with a clearer understanding of the functioning of muscle tissue through its fine time resolution. In topographical applications, magnetic domains and crystal structures can be studied with a precision and rapidity never reached before.

The specialized lectures on X-ray spectroscopy and crystallography, fluorescent analysis and on structural research on biological substances made the point very clearly that, with the advent of synchrotron radiation facilities, experimental physics has taken another leap forward.

In other sections of the general exhibition, five examples of modern instrumentation were presented, a reminder that industry is keeping pace with the high demands of the presentday experimental physicist. Information was given also on techniques developed for the remote measurement of physical parameters. Publishers showed that they cater for the increasing demand and supply of printed information which the publication explosion brings with it, and INSPEC of the Institution of Electrical Engineers provided a computer terminal with access to abstracts and reference data, a service appreciated by many visitors.

\section{Education and Popularization}

The laws of evolution require that attention be paid to the continuation of the species. Of course, this does not mean that physicists should think solely about the reproduction of the species of physicists, but the problem is none the less important. In the past, the problem of how to teach physics has in the main been the province of fanatics, but more and more it has become a matter of concern for physicists in general. It is significant that at York, the lecture by Prof. R. Fuller on this subject was included in the plenary session programme, and it was a matter of much regret that Prof. Jean Piaget did not feel well enough to participate. The Workshop on Physics Teaching was expected to attract about 30 participants but more than double asked to take part.

The main problem is to find a language in which we can talk about the teaching and learning of physics. Prof. Fuller, a dedicated and articulate disciple of Jean Paget, sought to illustrate his principles by involving his audience - a process that fascinated many but did not convince all. Piaget, without penetrating the methodology of physics, tries to explain the process of cognition and reasoning (understanding) of the physical world. As described in EN, 9
(1978) 4, p. 11, he identifies the following phases of cognitive development: sensory-motor, pre-operational, concrete-operational, formal-operational. They are described by the ability to solve certain physical problems, and are based on tests which appreciate the skill in a critical approach to physical observations. It is useful to recapitulate briefly these stages. The first, sensory-motor pre-verbal stage covers the first 18 months of life. During this stage, constructions such as the permanent existence of objects, temporal succession and elementary sensory-motor causality, are developed. The beginnings of language, of symbolic function of representation is attributed to the second stage: preoperational. In the third stage, actions appear which operate on objects, but not yet on verbally expressed hypotheses. These are operations of classification, ordering, the idea of number, etc., and even the fundamental operations of elementary logic of classes and relations, elementary mathematics and physics. The fourth stage: formal-operational, can be called hypothetic-deductive. In this stage the child can reason on hypotheses, not only on objects. This is the level on which completely abstract reasoning is possible.

The development of formal reason- ing should be an explicit aim of education, and the teaching of physics must conform with this scheme. It must also acknowledge the "boundary conditions" i.e. it must be adapted to the phase of cognitive development of the students and must provoke their further development in a proper way.

Contrary to the traditional technique of "pouring knowledge into heads", the only natural ways of teaching are the so-called active methods. They are characterized by the following factors:

autonomy - no strict control over the activity of students

\section{Job Opportunity for an \\ Experimental Physicist}

There is an opening for a post-doc or more senior scientist at the Labor. Für Hochenergiephysik der Eidgenössischen Technischen Hochschule (ETH) in Zürich. Experimental physictsts who are interested in doing and planning medium energy particle physics experiments at the Schweizerische Institut für Nuklearforschung (SIN) should write to :

Dr. P.G. Seiler

Laboratorium für Hochenergiephysik

Eidgenössische Technische Hochschule c/o SIN

$\mathrm{CH}-5234$ Villigen / Switzerland 